\title{
Methicillin-Resistant Staphylococcus aureus (MRSA) Detection from the Hands of Jatinangor Community Health Center's Health Care Providers
}

\author{
Jeevanisha Patmanathan, ${ }^{1}$ Sunarjati Sudigdoadi, ${ }^{2}$ Ricky Adriansjah ${ }^{3}$ \\ ${ }^{1}$ Faculty of Medicine Universitas Padjadjaran, ${ }^{2}$ Department of Microbiology and Parasitology \\ Faculty of Medicine Universitas Padjadjaran, ${ }^{3}$ Department of Urology Faculty of Medicine \\ Universitas Padjadjaran/Dr. Hasan Sadikin General Hospital Bandung
}

\begin{abstract}
Background: Methicillin-resistant Staphylococcus aureus is a bacterium that is resistance towards $\beta$-lactam antibiotics, and it seems to be one of the leading causes of nosocomial infections. Hands of the health care workers are said to be the main source for the nosocomial transmission. Thus, the study aims to determine methicillin-resistant S. aureus from the hands of Jatinangor Community Health Center's health care workers. Methods: Samples were taken from the hands of 30 Jatinangor Community Health Center's staffs, including medical and paramedical; from October 2012 to November 2012. Then, these samples underwent further laboratory examinations, starting from culture, identification and susceptibility test towards cefoxitin, in identifying methicillin-resistant $S$. aureus.

Results: Out of the 30 samples taken, 6 samples (20\%) were positive for S. aureus isolates. In which, 4 $(13.33 \%)$ of the samples were positive for methicillin-resistant S. aureus.

Conclusions: Since, health care workers are the main people in contact with patients and maintaining proper hand hygiene makes a huge difference; hand hygiene should be given adequate attention for the benefit of all. [AMJ.2015;2(1):217-20]
\end{abstract}

Keywords: Hand hygiene, health care workers, methicillin-resistant Staphylococcus aureus, nosocomial infection, Staphylococcus aureus

\section{Introduction}

Staphylococcus aureus (S. aureus) is one of the common strains of staphylococci. It can be found as a colony of the normal flora of the skin and the mucus of the nose. A person who is colonized by S. aureus can be asymptomatic, until it invades the body system and causes an infection. Staphylococcal infection can cause minor problems such as skin disease to major problems such as pericarditis that can be deadly. However, these problems can be overcome easily by giving antibiotics. ${ }^{1}$

As years pass by, $S$. aureus has developed resistance towards antibiotics, starting from penicillin, then methicillin and more recently towards vancomycin. This feature of the bacteria makes it more difficult to be treated and hazardous to the ones whom are infected by it. Methicillin resistant S. aureus (MRSA) is one of the strains, where it can be either a hospital acquired infection or a community acquired infection. Hospital acquired infection is an infection acquired by the person due to or favored by the healthcare environment. Thus, this infection concerns the most, referring to the fact that people who are admitted or coming to the hospital are already ill, which makes them more vulnerable to this sort of infections. ${ }^{2}$

According to $\mathrm{CDC}$ statistical data, the rate of MRSA infection has been decreasing for the past decade in general. ${ }^{3}$ However, according to WHO Europe- Hospital acquired infection: The European content, hospital acquired infection still covers 4 to $16 \%$ in these regions. ${ }^{4}$ In addition to that, according to a research conducted in Indonesia itself, there is $22.9 \%$ of MRSA present among specimens obtained from the in-patients. Proving that, it is still an issue that has to be taken into consideration. ${ }^{5}$

The MRSA can be easily transmitted from person to person either by direct contact with 
the person who is infected, a carrier or by touching contaminated things or substances. According to researches done, healthcare workers play a major role in the transmission of MRSA. As they are the people who are mainly in contact with the patients, in every possible step or procedure a patient undergoes in the healthcare settings. Moreover, a healthcare worker has to handle their patients, somehow with hands. Practically, every clinical procedure involves the usage of hand. Thus, hand seems to be the main mode of transmission among the health care workers. ${ }^{4,6}$

In addition to the fact that hand is commonly colonized by the bacteria which are normal flora; one can contaminate his hand by touching a patient or things that are contaminated with bacteria and cause cross transmission as this could be dangerous. ${ }^{4,6}$ That is the reason why hand hygiene is a major protocol that should be followed in eradicating this problem; and studies prove that hand hygiene compliance have improved infection rates in hospitals; even though it differs from one health care setting to another and needs to be monitored closely. Thus, this study is conducted to evaluate if MRSA can be found from the hands of healthcare workers of Jatinangor Community Health Center.

\section{Methods}

An observational study using the descriptive statistical method was done at Jatinangor Community Health Center from OctoberNovember 2012. Meanwhile, the lab examinations were carried out in Microbiology Laboratory of Faculty of Medicine, Universitas Padjadjaran (UNPAD).

Medical and paramedical of Jatinangor Community Health Center, were studying the population during the period of study. Thirty samples of hand swabs were taken from them. The samples were selected using probability sampling method which was a simple randomization. The inclusion criteria for the samples were staffs of Jatinangor Community Health Center who were in contact or involved directly or indirectly with the patients. Whereas, the exclusion criterion was staffs that already had an existing skin disorder or clinical conditions.

The experiment contains 3 main steps; sample collection and isolation, S. aureus identification and susceptibility test towards cefoxitin.

At first, hand swabs were taken by rolling the moisten cotton swab over the hand areas of the samples. The swabs were then transferred into the tube containing tryptic soy broth, which was the transport media; to be transported to the laboratory. The subjects were then asked to answer and performed 2 questions related to hand hygiene, that were in the inform consent form. ${ }^{7}$

Next, the swab was cultured onto a mannitol salt agar plate using an inoculating loop and left to incubate overnight at a temperature of $37^{\circ} \mathrm{C}$. Staphylococcus aureus formed a yellowish colony. ${ }^{7}$ Then, bacterial smear was performed from the culture plate and gram staining procedure was carried out on it. The interpretation of the result was considered to be gram positive if it turned into purple colour; or gram negative if it turned into pink colour. Gram positive cocci indicated S. aureus, which can be seen as clusters under the microscope. ${ }^{7}$

Afterwards, bacterial smear was performed from the culture plate and catalase test was carried out on it. Where, an inoculating loop was used to take a small amount of colony from the agar plate and mixed gently with the 3\% hydrogen peroxide solution on the glass slide. The slide surface was then closely observed for any immediate formation of gas bubbles. Immediate gas bubble formation indicated a catalase positive result. If there were no gas bubbles or few scattered bubbles released then it was considered as a catalase negative result. Staphylococcus aureus gave a catalase positive result. 8,9

The procedure was then continued with coagulase slide and tube test. Where, for the coagulase slide test, bacterial smear was performed first. Then, an inoculating loop was used to take a small amount of colony from the agar plate and mixed gently with the human plasma on the glass slide, if clump formed after 5-10 seconds, then it was considered coagulase positive result. If there was no clump formation then, it was considered coagulase negative result. For a slide negative result, we had to proceed by performing a coagulase tube test. ${ }^{8,9}$

For the coagulase tube test; using an inoculating loop, a small amount of colony was taken from the culture sample and transferred into a test tube containing $0.5 \mathrm{ml}$ of human plasma; and mixed together. The test tube was then incubated at $37^{\circ} \mathrm{C}$ for about 4 hours. It was checked if the content had coagulated or solidified, every 1 hour to 4 hours. It indicated a coagulase positive result, if the content had solidified and it was a coagulase negative result, if the content did not solidify. 
Finally, susceptibility test against cefoxitin was performed. The swab was streaked onto the Mueller-Hinton agar; covering all area including edges of agar plate and left to dry. One cefoxitin disc was placed at the center of the inoculated surface by using a forceps. The cefoxitin disc was then pressed against the plate gently, using a needle. The agar plate was then placed in an incubator at $37^{\circ} \mathrm{C}$ for 24 hours. After 24 hours, the diameter of the zone was measured from the bottom of the plate by using a ruler in millimeter and the result was interpreted according to Clinical and Laboratory Standard Institute (CLSI) zone diameter interpretive standards for Staphylococcus sp. Where, when we used a $30 \mu \mathrm{g}$ cefoxitin; if its diameter was $\geq 22 \mathrm{~mm}$ it was susceptible and if its $\leq 21 \mathrm{~mm}$ it was resistance. ${ }^{10,11}$

\section{Results}

Histopathological appearance of liver biopsy cThe research was conducted by obtaining 30 samples randomly at the times of visit, which was usually at the peak hours of work (9am$12 \mathrm{pm})$. The hands were swabbed and the samples were then taken to the Microbiology Laboratory of Faculty of Medicine of UNPAD Jatinangor for further microbiological examination to identify the presence of MRSA

In addition, out of the 30 samples that were asked on their awareness on existence of proper hand-washing technique, 28 of them $(93.33 \%)$ acknowledged that they knew about it. However, out of these 30 samples, only 12 of them (40\%) managed to perform the handwashing steps in an acceptable manner.

\section{Discussion}

Based on table 1, about $20 \%$ of the samples obtained are positive for S. aureus; which is not considered abnormal because S.aureus is usually found on the normal skin flora of humans. In addition, it does not cause any harm to the carrier until it manages to get into the body system via an open wound or cut, where it starts to be infectious.

However, around $13.33 \%$ of the samples obtained are positive for MRSA. MRSA is a strain of $\mathrm{S}$. aureus that is resistance towards $\beta$-lactam antibiotics. In fact, it has the tendency to develop resistance towards other type of antibiotics. One of the main reasons for the development of this resistance is the excessive usage of antibiotics which occurs very much in a health care settings nowadays. As a consequence, MRSA is more difficult to be treated.

The MRSA which was found among the hands of the health care workers; may be due to the pattern of transmission. As it can be transmitted from a colonized person to a noncolonized person or contaminates the things in the surroundings which could come into contact with a person somehow and cause colonization. Being colonized by MRSA is not dangerous until it manages to get into our body system and cause an infection. MRSA infection could be deadly at times as statistics shows that thousands have died of it yearly. That is why MRSA colonization has to be taken into high consideration, even before it becomes infectious. Based on a previous study, 7.1\% of the health-care workers were positive MRSA on their hand swabs. This proves that hand is one of the vehicles for MRSA transmission. ${ }^{12}$

One of the easiest steps to control the transmission of MRSA is by hand-washing, and when the samples were asked on their awareness of proper hand-washing technique $93.33 \%$ of them claimed to know it. However, when they were asked to perform the steps that they claim to know, only $40 \%$ of them managed to perform in an acceptable manner while other claimed to have forgotten them. This showed the lack of awareness on the importance of hand cleanliness.

That is why hand-washing has received a great attention among many recently. There are many hand-washing campaigns that are being launched nowadays. For instance, the one launched October 2011 in Jakarta by the Ministry of Health of Indonesia entitled

Table 1 Percentage of Methicillin-resistant Staphylococcus aureus (MRSA) from Isolates of the Hand Swabs

\begin{tabular}{lcc}
\hline \multicolumn{1}{c}{ Bacteria } & Number of samples & Percentage \% \\
\hline S.aureus (+) & $6(30)$ & 20.00 \\
MRSA (+) & $4(30)$ & 13.33 \\
MRSA (+) from S.aureus (+) & $4(6)$ & 66.67 \\
\hline
\end{tabular}


'Indonesia Welcomes Sanitation Month' and a yearly celebration of 'Hand Washing with Soap Day' that holds on every 15 October in Indonesia. Everyone should take this opportunity and participate in it. ${ }^{13}$ Thereby, more attention should be paid by every health care centers on their hand-washing protocols; as it is the key to infection-control.

Over the last decade improved hygiene has caused a drop in the MRSA cases globally. In fact, in a 9 year study conducted by Virgina Commonwealth University, revealed that hand-washing in conjugation with other simple hygiene measures, reduces the risk of MRSA infection by $95 \%$ in ICU. This just proves the importance of hand-washing compliance. ${ }^{14}$

Based on this study the researcher can conclude that there is a presence of MRSA among the health care workers of Jatinangor Community Health Center. However, further research should be done to find out the real cause behind the contamination and methods to improve them.

\section{References}

1. Harvey RA, Champe PC, Fisher BD. Microbiology. Philadelphia: Lippincott Williams \& Wilkins; 2006. p. 69-77.

2. Ji Y. Methicillin-Resistant Staphylococcus Aureus (Mrsa) protocols. Totowa, New Jersey: Humana Press; 2007. p. 1-20.

3. Behen M. Hospital-acquired MRSA infections on the decline, CDC says. New Jersey: Bloomberg Businessweek; 2010 [downloaded in 4 May 2012]. Available at: http://www.businessweek.com/lifestyle/ content/healthday/642013.html.

4. WHO. WHO guidelines on hand hygiene in health care. Georgia: WHO; 2009 [downloaded in 7 May 2012]; Available at: http://www.safecarecampaign.org/HandHygiene_files/WHOHandHygieneGuide. pdf.

5. Parwati I, Turbawaty DK, editor. Peta bakteri dan kepekaannya terhadap berbagai antibiotika di Rumah Sakit Umum Pusat Dr.Hasan Sadikin Bandung Semester II Tahun 2010. 1st ed. Bandung: Patologi
Klinik Rumah Sakit Umum Pusat Dr.Hasan Sadikin; 2011. p. 27.

6. CDC. Guideline for hand hygiene in health care settings. Atlanta: CDC; 2002; 51 [downloaded in 29 April 2012]. Available at: http://www.cdc.gov/mmwr/pdf/rr/ rr5116.pdf.

7. Isenberg HD. Essential procedures for clinical microbiology. Washington, DC: ASM Press; 1998. p. 1-36.

8. Swamy PPM. Laboratory manual on biotechnology. New Delhi: Rastogi Publications; 2008. p. 256.

9. Estridge BH, Reynolds AP. Basic clinical laboratory techniques. New York: Cengage Learning; 2011. p. 761-74.

10. Vandepitte J, Engbaek K, Piot P, Heuck CC. Basic laboratory procedure in clinical bacteriology. Geneva: WHO; 2003 [downloaded in 2 May 2012]; 2nd:[Available at: http://whqlibdoc.who. int/publications/2003/9241545453.pdf.

11. CLSI. Performance standards for antimicrobial susceptibility testing; Twentieth Informational Supplement. Wayne, PA: Clinical and Laboratory Standards Institute; 2010. p. 70.

12. Rosyati DOF. Pemeriksaan Staphylococcus aureus resisten Metisilin (MRSA) pada perawat bagian RSUP Dr Hasan Sadikin Bandung [Thesis]. Bandung: Universitas Padjadjaran; 1998.

13. Faizal EB. Government launches hand washing campaign. Jakarta: The Jakarta Post; 2011 [downloaded in 25 November 2012]. Available at: http://www. thejakartapost.com/news/2011/10/10/ govt-launches-hand-washing-campaign. html

14. Labs PM. Handwashing may be the most effective way to reduce MRSA infections. Dickson: Private MD Labs; 2012 [downloaded in 20 November 2012]. Available at: http://www.privatemdlabs. com/blood-testing-news/Infectious_ Diseases/Hand-washing-may-be-themost-effective-way-to-reduce-MRSAinfections \$800892993.php. 